\title{
Local Binary Pattern Method (LBP) and Principal Component Analysis (PCA) for Periocular Recognition
}

\author{
Sereen Alkhazali ${ }^{1}$, Mohammad El-Bashir ${ }^{2}$ \\ Department of Computer Science \\ Faculty of Computer Science and Information Technology \\ Al-al Bayt University - Jordan
}

\begin{abstract}
Identification of identity through eye is gaining more and more importance. Commonly, the researchers approach the eye from any of three parts, the iris, the circumference around the eye, and the iris and its circumference. This study follows a holistic approach to identity identification by using the iris and whole periocular area and proposes a periocular recognition system (PRS) that has been developed using the Local Binary Pattern (LBP) technique combined with Principal Component Analysis (PCA) at the feature extraction stage and the k-nearest neighbors (k-NN) algorithm as a classifier at the classification stage. This system achieves identity recognition through three steps: pre-processing, feature extraction, and classification. Pre-processing is applied to the images so as to convert them to grayscale. In the feature extraction step, the LBP method is applied to extract the texture feature from the images and use it in PCA to reduce data dimensionality and obtain the relevant data so that only the important features are extracted. These two steps are applied both in the training phase and the testing phase of image processing. On the other hand, the testing data sets are processed using the k-NN classifier. The proposed PRS was tested on data drawn from the PolyU database using more than one basis of system experience. Specifically, the system performance was tested once on all 209 subjects present in the database and once on 140 subjects. This database also contains images taken in the visible (VIS) and near-infrared (NIR) regions of the electromagnetic radiation (EMR) spectrum. So, the system was tested on images taken in both regions separately for matching. As well, the proposed PRS benefited from the availability of images for the right and left perioculars. Performance was, therefore, tested on images of each side of the periocular area (the left and right sides) separately, as well as for the combination of the two sides. The identity recognition rates characteristic of the proposed PRS were most often higher than the recognition rates produced by systems reported in the literature. The highest recognition accuracy obtained from the

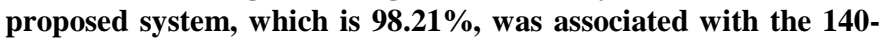
subject data sub-set.
\end{abstract}

Keywords-Periocular recognition; Local Binary Pattern (LBP); Principal Component Analysis (PCA); k-Nearest Neighbors $(k-N N)$

\section{INTRODUCTION}

Nowadays, security of systems is more important than the systems themselves and authentication serves as the first line of defense against any unauthorized persons. Most frequently, authentication can be achieved by one of three ways: password, biometry, and a characteristic identifier of the user such as a card. Biometry provides secure means of authentication and identification owing to that it is difficult to steal, or even replicate, biometric information. Furthermore, many options are available for biometric identification such as fingerprint, DNA, sound pattern, signature, iris patterns, periocular print, and retina patterns. As far as face recognition is concerned, the periocular print is the most accurate biometric property that can be used for face identification [1].

Periocular authentication is an automated method of biometric identification that applies mathematical pattern recognition techniques on the video images of one or both of the eyes of a person, whose complex patterns are unique, stable, and can be seen from some distance. According to Kumari and Seeja [2], authentication based on the periocular region builds on features taken from both the face and iris. In this context, the periocular biometric expresses the facial region right close to the eye [2], and the periocular region, which is the area around the eye, encircles the eyebrows, eyelashes, eyelids, and the adjacent skin area [3]. But nlike acquisition of many other ocular biometrics, acquisition of a periocular biometric does not demand a high user cooperation and a close capture distance [4]. However, even though the periocular area is regarded as highly-discriminating part of the human face, its utility as independent soft biometric or modality is still under scrutiny. Hence, it is a research goal to establish performance metric for features of the periocular area so that their likely use in combination with the face or iris can be assessed.

The periocular recognition system (PRS) is a system designed for identification of people through the iris and the region surrounding the eyes, which is a region that provides wide space and feature richness for discriminating people and identifying the authorized person. Within this context, the major objective of this study was to develop a PRS that can effectively recognize people through their eyes. After acquisition of cross-spectral iris data (e.g., from the PolyU Cross-Spectral Iris Database), the proposed PRS processes the images in three steps: (i) converting the original image to the grayscale; (ii) extracting the features by using the Local Binary Pattern (LBP) method and Principal Component Analysis (PCA); and (iii) classifying the extracted features using the knearest neighbors (k-NN) algorithm. 


\section{LITERATURE REVIEW}

Many classifiers have so far been employed for recognition of the periocular iris. They include varied Artificial Intelligence (AI) systems like the SVM, Histogram of Oriented Gradients (HOGs), and the Feed-Forward Back Propagation Neural Network (FBPNN), amongst others.

Woodard et al. [5] illustrated application of the fusion methods on iris and periocular images in the case of non-ideal images of the eye, namely, images that are distinguished with poor contrast, occluded irises, illumination artifacts, and motion and spatial blur. Outcomes of their analysis indicated that using the Multi-Biometric Grand Challenge (MBGC) database with score level fusion can enhance recognition performance in such images. Periocular texture is extracted from small, fixed region of the skin that surrounds the eye. Experiments on the images extracted from the near infra-red (NIR) face videos of the MBGC dataset using the fusion techniques demonstrated that valuable information is contained in the periocular area and that it can be integrated with the iris texture data to enhance the overall identification accuracy in atypical conditions.

Periocular biometrics include valuable information for the iris and face recognition systems. Fasca et al. [3] developed a PRS that employed the HOGs and LBP method for extraction of features from periocular images. The LBP is a kind of features that is employed for classification in capturing vision. It is a powerful feature for the texture. In other respects, the HOGs were employed for the purpose of object detection using gradient features. For effective recognition and classification of authorized personnel, these researchers [3] used the back propagation neural network (BPNN) classifier. Eventually, the PRS developed by these researchers had a recognition accuracy of $91 \%$.

The first algorithm based on the Artificial Neural Network (ANN) for characterizing and classifying the image variations caused by different spectral ranges was proposed by Sharma et al. [6]. The algorithm employed the ANN for distinguishing the variabilities generated by two spectra; the VIS and NIR regions of the EMR spectrum. First, the spectra were trained individually and, then, joined such that, by the use of crossspectral training data, the proposed algorithm can learn the cross-spectral variations. For evaluation of performance, a cross-spectral periocular database was prepared that consists of NIR and VIS night spectra images. The database was the IIITD multispectral periocular (IMP) database that includes 1,240 iris images of 62 individuals and which were captured within the VIS spectrum, night vision, and NIR iris cameras. The evaluation results pointed out that this proposed approach produced much higher levels of accuracy than four methods (Pyramid of Histograms of Oriented Gradients (PHOG), HOG, FPLBP, and LBP). The concomitant recognition rates ranged from $76.97 \%$ for the visible spectrum to $92.5 \%$ (NIR) in the "same-spectrum" analysis and from $48.21 \%$ for the night vision NIR to $71.93 \%$ for the visible night vision in crossspectral analysis. In most experiments, combined left and right $(\mathrm{L}+\mathrm{R})$ periocular recognition had higher accuracy than single left or right $(\mathrm{L} / \mathrm{R})$ recognition.
Nie et al. [7] clarified that accurate automatic biometric identification of identity by using periocular imaging had broad spectrum of applications, extending from human surveillance to enhancement of performance for the IRSs, particularly under less-constrained imaging environments. The Restricted Boltzmann Machine (RBM) is a generative stochastic ANN that has the ability to learn the probability distribution of its input data. The Convolutional Restricted Boltzman Machines (CRBM) aim at accommodating large image sizes and markedly reducing the computational burden. However, the methods of unsupervised learning of features had not been investigated in the biometrics domain, except in face recognition. These researchers [7] explored effectiveness of the CRBM in periocular recognition. They implemented experiments on a publicly-available database of periocular images of a large sample of subjects (300 test subjects), namely, the Ubripr database, and exploited key features simultaneously to improve the matching accuracy. The results of experiments confirmed effectiveness of RBM feature learning in automated periocular recognition with large numbers of subjects. In addition, their results suggest that supervised metric learning can result in better identity recognition than the Euclidean distance.

Behera et al. [8] underscored that periocular recognition had been active domain of research in the last few years. They suggested an identity recognition method that is based on illumination normalization of NIR and VIS periocular images. Their proposed method entailed normalization of the images by using the difference of Gaussian (DoG) filtering, followed by calculation of descriptor that captured the structural details present in the illumination-normalized images by using the HOG. Lastly, those feature vectors that correspond to the query and the enrolled image were compared based on the cosine similarity metric so as to produce a matching score. Performance of this algorithm was, then, assessed on three publicly-available benchmark databases of cross-spectral periocular images.

Ramaiah et al. [9] developed an identity recognition method based on Markov random fields (MRFs) and threepatch LBP (TPLBP) descriptor. They used exact pixel correspondences during image acquisition and synthesized the NIR image pixels from the VIS periocular images. Two versions of LBP descriptors, that is, the TPLBP and four-patch LBP (FLBP) were then extracted from the images. The method was then evaluated on the IMP and PolyU datasets using both descriptors. The evaluation results pointed out that the TPLBP descriptor outperformed the FLBP operator as it had Guanine Accept Rate (GAR) values of $18.35 \%$ and $73.20 \%$ at $1.0 \%$ FAR when tested on the IMP and PolyU databases, respectively.

\section{MethodOLOGY}

The periocular recognition system (PIRS) progresses in four sequential steps as shown in Fig. 1. The first step is acquiring image by camera. The second step is preprocessing, whereby the original image is converted to a grayscale image. The third step is extracting features, whereby both the LBP method and PCA are employed in order to extract the most important features for recognition. The last step is classifying 
the extracted features, in which a comparison is held between a new biometric feature and one model or all models in the database. This step outputs a similarity score for every comparison by using the k-NN classifier. Then, performance analysis is conducted so as to determine whether or not the probe and the model that are obtained from the database match on the basis of whether the similarity score is higher or lower than a pre-set match threshold.

Sometimes, methods of transforming the raw feature vectors, i.e., feature extraction methods, are employed before application of the pattern-matching algorithm. The algorithms of feature extraction try to transform a large-dimensionality feature space to a smaller-dimensionality space that is easier to process and which encodes less redundancy by use of mathematical methods like the LBP method and PCA.

\section{A. Database}

The PolyU Cross-Spectral Iris dataset is a distinctive, bispectral dataset of iris images that was developed for crossspectral iris recognition studies and made publicly available. This dataset is a compilation of face images taken under the condition of simultaneous bi-spectral imaging, from both the left and right eyes. A sample of such images is given in Fig. 2. Overall, this dataset is made up of a total of 12,540 iris images $(209 \times 2 \times 2 \times 15)$ that were obtained from 209 subjects in 15 instances, each. Every one of these iris images has the dimensions of nearly $640 \times 480$ pixels. Moreover, the pixel correspondences of these iris images lie both in the VIS and NIR regions of the EMR spectrum [10].

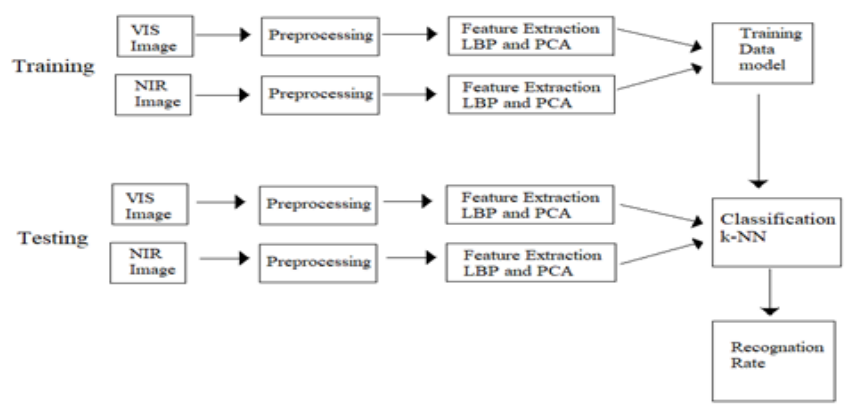

Fig. 1. General Structure of the Periocular Recognition System (PRS).

Bi-spectral (Right Eye)

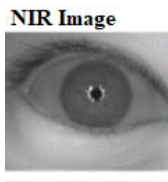
VIS Image
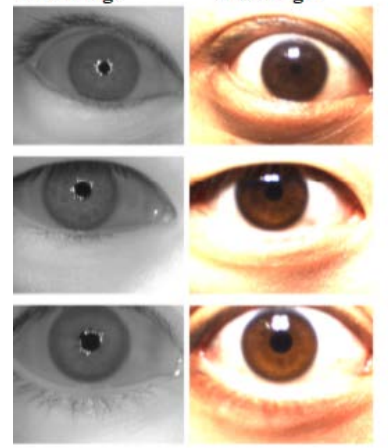

Fig. 2. Sample Iris Images Simultaneously Shot in the NIR and VIS Regions of the EMR Spectrum with Full Pixel Correspondences (Source: [10]).
It should be highlighted that the researchers encountered several difficulties in the present study while working with this database, the most critical of which was that the related previous studies did not specify which particular data of this dataset they used. This prompted the researchers to expand the current inquiry in an effort to grow able to make reasonable comparisons with relating previous studies.

\section{B. Periocular Recognition System (PRS)}

This study develops a PRS that is based on a combined approach to feature extraction and the k-NN classifier. This proposed system achieves periocular recognition in three steps as explained in the subsequent sub-sections.

\section{1) Preprocessing}

The proposed PRS handles the inherent variations in illumination between the NIR and VIS images in the preprocessing phase by converting the images to the gray scale. Variations in illumination impact matching of the VIS periocular images negatively. Every image will be transformed into binary values and stored in a matrix for later evaluation.

After converting the periocular image to a grayscale image in the pre-processing step, it is analyzed for each person and features are, then, extracted using the combined LBP-PCA recognition system and stored in a log file in the database for verification and authentication. Upon receipt of an image for authentication, features are analyzed and extracted by using the combined system and a new record for comparison is created in the next (classification) step. A description of periocular image feature extraction is given in the subsequent sections.

The LBP method is very useful because it is a robust method for illumination of change. It is a sort of visual descriptor that is employed for classification in computer vision and which proved to be powerful tool for texture classification. It improves the detection performance and is simple and quick to compute. However, certain binary patterns exist more commonly in some texture images than others. In its simplest form, the LBP feature vector is created in the following manner: divide the periocular image into cells (e.g., $3 x 3$ pixels for each cell), then encode the center pixel by a series of bits for each center pixel in a cell of eight neighbors. The neighboring pixels are given a binary value of 1 if they are equal to, or higher than, the central pixel and 0 if otherwise. Eight-bit binary number is then formed by concatenating the bit. Accordingly, its decimal equivalent becomes the label for the center pixel, which is denoted by a circle as shown in Fig. 3.

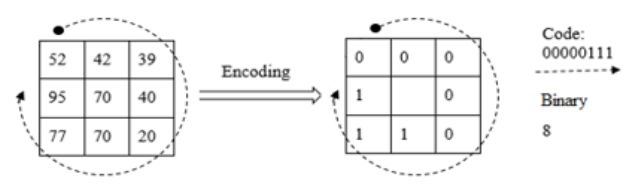

Fig. 3. Example of LBP Encoding.

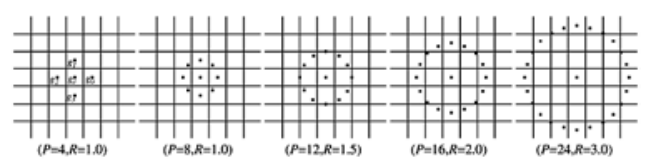

Fig. 4. Symmetric Circular Neighbor Sets for Different P and R Values (Source: [11]). 
This study employed the LBP features in an effort to analyze texture of the entire image for eye recognition. The fundamental LBP method takes into account small circularity neighborhood and a rotation invariant texture which has symmetric points $(\mathrm{P})$ that are pixels spaced equally on circle of a radius (R) for the whole eye image by use of a global threshold. Fig. 4 shows some sizes and shapes of eye images produced by the LBP method.

\section{2) Principal Component Analysis (PCA)}

Principal Components Analysis (PCA) is a standard, and practical, statistical tool for data analysis that has established applications in various areas like neuroscience, face recognition, and image compression [12].

In this analysis, the first component that is extracted (Component 1) explains the maximum amount of the total variance in the observed variables. Under the ideal conditions, this implies that the first component correlates the highest with some observed variables. Though, however, it may correlate with many. The second extracted component (Component 2) has two important characteristics. First, it accounts for the maximum amount of the variance in the data that was not explained by the first component. Again, under the ideal conditions, this suggests that the second component correlates with some of the observed variables which do not show high correlations with Component 1 . The second characteristic of Component 2 is that it does not correlate with the first component. That is, if one is to assess correlation between the first two components, then the correlation coefficient is zero. The rest extracted components exhibit the same two characteristics; every component explains the maximum amount of the variance in the observed variables that is not explained by the previous components and is not correlated with any previous component. Principal component analysis progresses in this way, with every new component explaining progressively lower and lower proportion of variance. This is why usually only the first few components are kept and interpreted. Once the analysis is complete, the extracted components manifest varying degrees of correlation with the observed variables, but are entirely uncorrelated with one the other [12].

The main steps in PCA are the following:

Step 1: Getting relevant data

In this study, a 2x2 data sub-set is drawn from pixel map of the image.

\section{Step 2: Subtracting the mean}

In PCA, one should subtract the mean from every data dimension. The subtracted mean is the average across every dimension. Hence, all the $\mathrm{x}$ values have the mean of the $\mathrm{x}$ values of all data points deducted from them. Likewise, all the $\mathrm{y}$ values have the mean of all the data points deducted from them. This generates a dataset with a mean of zero.

Step 3: Computing the covariance matrix

The covariance matrix is mainly created for the following two purposes: i) Assessing the extent to which every dimension varies from the mean.

ii) Estimating the covariance between two dimensions to determine whether or not there are relations among them.

It should be underlined that covariance between any dimension and itself is, in fact, the variance.

In this regard, the following equation Eq. (1) was employed to compute the covariance of the two dimensions:

$\operatorname{Cov}(\mathrm{X}, \mathrm{Y})=\frac{\sum_{\mathrm{i}=1}^{\mathrm{n}}(\overline{\mathrm{X}}-\mathrm{X})(\overline{\mathrm{Y}}-\mathrm{Y})}{(\mathrm{n}-1)}$

The diagonal of the covariance matrix represents variances of $x, y$, and $z$, e.g., $\operatorname{cov}(x, y)=\operatorname{cov}(y, x)$. Thus, the matrix is symmetrical about the diagonal element. The resultant covariance may be categorized into three types:

i) Positive covariance when the two dimensions decrease or increase simultaneously.

ii) Negative covariance when one dimension decreases and the other increases,

iii) Zero covariance when the two dimensions are independent.

The covariance is calculated to define the relations among dimensions in high-dimensional data (usually higher than three) where visualization is difficult.

\section{3) Classification}

Classification is a process which recognizes a periocular image by finding a match of its features with one of the periocular images in the dataset. The PRS proposed in this study went through two phases: a testing phase and a training phase. The classifier was used only in the testing phase to identify a periocular image via its features. The features of the periocular image were extracted in the testing phase by the combined LPB-PCA system according to the same steps followed in the training phase. Then, the features of the training model were matched so as to identify the unknown image.

The k-NN algorithm is a simple algorithm that stores the available cases and classifies the new ones based on some similarity measure. It is one of the predictive models and it does not need to learn complicated mathematical equations; it just needs to (i) have a way for calculating the distance between data and (ii) perform a hypothetical investigation to ensure that the data close together are similar and far away from others [13].

Most of the techniques used in the predictive model look at the dataset as a whole in order to characterize the data patterns, but the nearest neighbors conceal a lot of information because they are predicted for each new point depending only on the number of points near it.

The output of the k-NN algorithm is a label of the closest owner of the testing periocular image. The particular object is classified by means of plurality vote of its nearest neighbors, with the object chosen by sorting all objects in descending order of votes and selected from its k nearest neighbors, where 
$\mathrm{k}$ is a positive, typically, small, integer. This study adopted the $\mathrm{k}$ value of 5 .

The main steps of the k-NN algorithm are the following:

- Determine the value of the variable $\mathrm{k}$, which is the number of neighbors.

- Compute distances between the new image and the images in the dataset.

The distances between the testing and training images ( $p$ and q) in the dataset are calculated using the equation (2):

$d(p, q)=\sqrt{\sum_{i=1}^{n}\left(p_{i}-q_{i}\right)^{2}}$

- Arrange the images to get the neighbors based on the lowest distance calculated in the previous step, and take the number of the neighboring ks.

- Define a label for the neighbors.

The label, namely, the vast majority of neighboring images, is the expected label by Eq. (3)

$d(p, q)=\sqrt{\sum_{i=1}^{n}\left(p_{i}-q_{i}\right)^{2}}$

Thereafter, the recognition rate (accuracy, in effect) is calculated by using the following equation Eq. (4):

$R R=\frac{\text { correctly detected periocular images }}{\text { Total no.of periocular image }} * \mathbf{1 0 0}$

\section{4) Implementation}

Practically, the proposed PRS was run on PC to examine its performance that has Intel (R) Core (TM) i5-8265U processor with CPU@2.20 GHz, fitted with 4 GB RAM, and operating on the Windows 8 operating system. The algorithms were written and executed in the Matlab 2018a programming language. However, some calculations were performed using Microsoft Excel 2010.

\section{RESUlTS AND DisCUSSION}

This study develops and proposes a PRS based on feature extraction with LBP and PCA. The features are fed into the kNN algorithm for classification. The proposed system was tested on the PolyU Cross-Spectral Iris dataset and its performance was compared with levels of performance of contemporary PRSs based on the recognition rate. This section presents the experimental results of this system in all cases and compares them with results of previous related works.

\section{A. Training and Testing}

To evaluate the identity recognition efficiency of the proposed PRS, this system was tested on the PolyU CrossSpectral Iris dataset of Hong Kong Polytechnic University. This dataset of periocular and iris images was built by drawing data from 209 subjects. Every single subject provided two sets of data. The first set comprises 15 images taken from the left side of the face whereas the second set contains 15 images taken from the right side of the face. In the present study, experiments were performed on iris images pertaining to 140 subjects. The first 10 images in each of the left and right data sets were used in training while the last five images in each of these two sets were employed in the testing. As well, the proposed PRS was tested using images taken in the visible light, both in the VIS and NIR regions of the EMR spectrum. On the other hand, training was conducted on images using near-inference (VIS-VIS and NIR-NIR) analysis and crossspectral (VIS-NIR) analysis.

\section{B. Experimental Result}

This section presents the results of testing the proposed PRS. To determine the conditions conducive to optimum classification, differing values of input parameters for feature extraction were examined. Then, the recognition results were compared with results of other systems based on the recognition rate. The analysis results are presented in two subsections, one each for:

i) The same spectral region.

ii) Cross-spectral regions.

1) Results Related to Images in the same Spectral Region

The PolyU dataset provides multispectral periocular images shot in the VIS and NIR regions of the EMR spectrum. In this sub-section, the researchers present the results of application of the proposed PRS to the same spectral regions in the training and testing steps, that is, the VIS-VIS and NIR-NIR regions.

The researchers chose to increase the parameters of the LBP $(\mathrm{P}, \mathrm{R})$ in order to get higher recognition accuracy than 95\%. The parameter $\mathrm{P}$ is a positive integer whose typical values range from 8 to 24 and the parameter $R$ is a positive integer, too, whose typical values range from 1 to 5 . The levels of performance of the suggested system in recognizing the 140 subjects taken from the PolyU dataset and shot in the VIS region are summarized by Table I and Fig. 5.

The evaluation results uncover that the recognition accuracy is higher for the radius value of 5 than the radius value of 3 and that the neighborhood value affects the recognition accuracy only slightly. The best recognition accuracy is associated with the $\mathrm{k}$ value of 18 (Table $\mathrm{I}$ and Fig. 5). In addition, this study tested performance of the proposed PIRS using data for the 209 subjects in the PolyU dataset using the same $\mathrm{P}$ and $\mathrm{R}$ values used in the case of the 140 subjects (Table I). The results indicate that the best accuracy of recognition (94.14\%) of the 140 subjects was associated with $\operatorname{LBP}(18,5)$.

TABLE I. PERFORMANCE OF THE PROPOSED PRS BASED ON DIFFERENT VALUES OF THE LBP PARAMETERS (P AND R)

\begin{tabular}{|l|l|l|l|}
\hline \multirow{2}{*}{ Neighborhood } & \multirow{2}{*}{ Dimensional } & \multicolumn{2}{l|}{ Recognition accuracy (\%) } \\
\cline { 3 - 4 } & histogram & $\mathrm{R}=1$ & $\mathrm{R}=5$ \\
\hline $\mathrm{P}=8$ & 59 & 92.50 & 95.01 \\
\hline $\mathrm{P}=16$ & 243 & 92.79 & 95.03 \\
\hline $\mathrm{P}=18$ & 309 & 92.50 & 95.14 \\
\hline $\mathrm{P}=20$ & 383 & 92.93 & 95.07 \\
\hline $\mathrm{P}=24$ & 555 & 92.50 & 94.93 \\
\hline
\end{tabular}




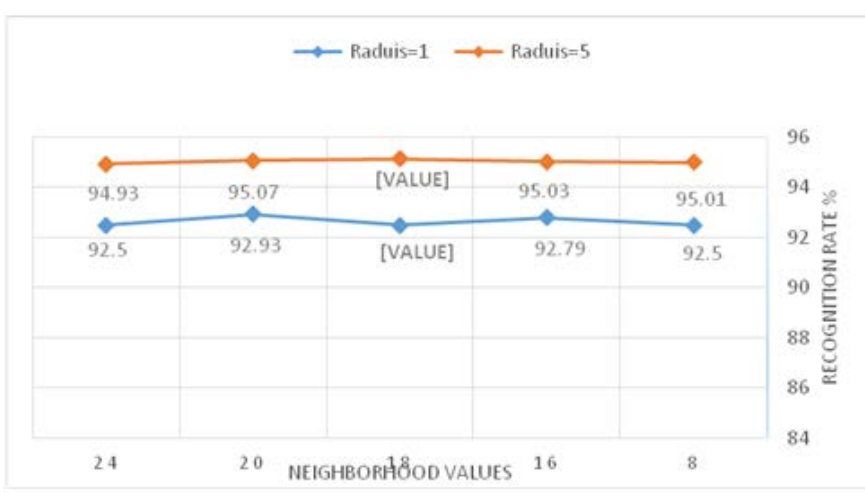

Fig. 5. Recognition Accuracy Values of the Proposed PRS based on Different Values of the LBP Parameters (P and R).

Table II displays the recognition accuracy values based on different bases, where the recognition accuracy was tested based on the number of images (209 and 140 subjects) used in matching by using the periocular images of right and left sides of the face, once separately, and once together. Lastly, levels of performance of the suggested PRS were examined for the two regions of the EMR spectrum separately, i.e., the VIS and NIR regions.

It is noted in Table II that the recognition rates are higher in all cases for the NIR region than for the VIS region since the former region relieves any physical discomfort resulting from the illumination, reduces the specular reflections, and increases the proportion of texture that is captured for some of the iris colors [14].

TABLE II. RECOGNITION ACCURACY VALUES FOR THE NIR AND VIS SPECTRAL REGIONS

\begin{tabular}{|l|l|l|l|l|}
\hline \multirow{2}{*}{ Side } & \multicolumn{4}{|l|}{ Recognition accuracy (\%) } \\
\cline { 2 - 5 } & \multicolumn{2}{|l|}{ VIS-VIS } & \multicolumn{2}{l|}{ NIR-NIR } \\
\cline { 2 - 5 } & 140 Subjects & 209 Subjects & 140 Subjects & 209 Subjects \\
\hline Left & 96.00 & 96.17 & 98.14 & 97.89 \\
\hline Right & 95.00 & 94.07 & 98.43 & 97.13 \\
\hline Both & 95.14 & 94.04 & 98.21 & 97.32 \\
\hline
\end{tabular}

In the case of images taken in the same spectral regions, the levels of performance of the proposed PIRS were the best in the case of the NIR-NIR owing to that the NIR region captures the details of the periocular area, even in the case of a heavilypigmented periocular image.

\section{Results of Cross-Spectral Analysis}

This sub-section highlights the results of the experiments performed on the cross-spectral periocular images. The PolyU dataset provides multispectral periocular images shot in the VIS and NIR regions. These images were processed in the training and testing steps much like the VIS-NIR images were.
TABLE III. RECOGNITION ACCURACY VALUES FOR BOTH THE NIR AND VIS SPECTRAL REGIONS

\begin{tabular}{|c|c|c|}
\hline \multirow{3}{*}{ Side } & \multicolumn{2}{|c|}{ Recognition accuracy (\%) } \\
\hline & \multicolumn{2}{|l|}{ VIS-NIR } \\
\hline & 140 Subjects & 209 Subjects \\
\hline Left & 100 & 100 \\
\hline Right & 99.93 & 99.95 \\
\hline Both & 97.07 & 96.41 \\
\hline
\end{tabular}

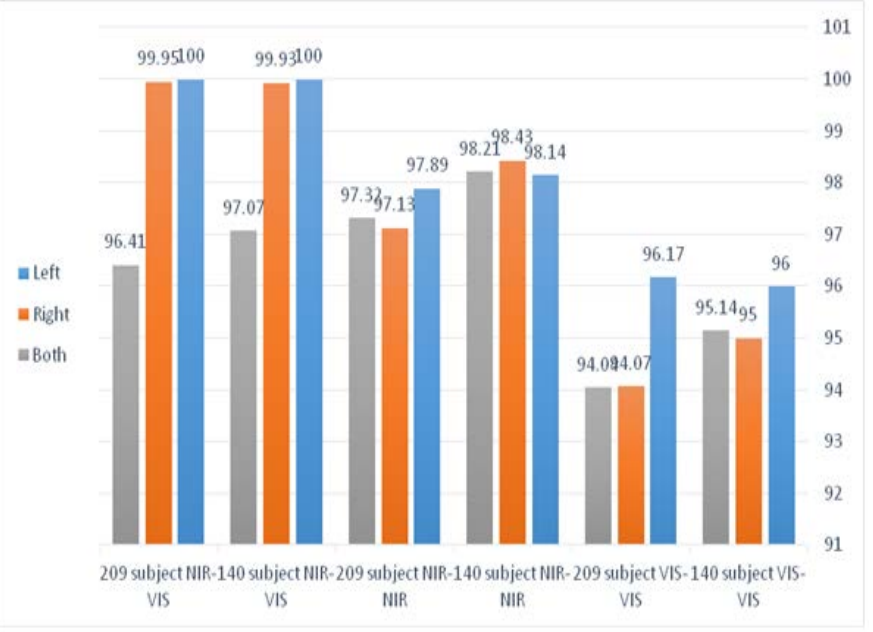

Fig. 6. Comparison of Recognition Percentages for both the VIS and NIR Spectral Regions and Cross-Spectral Analysis.

Table III shows the recognition percentages associated with cross-spectral matching for 140 and 209 subjects using single periocular images and pairs of periocular images. Additionally, Fig. 6 displays the results relating to all the cases of the EMR spectrum addressed by this study, including single periocular images and pairs of periocular images.

Fig. 6 unveils that the optimum recognition results were realized when matching was conducted for both the left and right periocular areas individually, then for their pairs. Moreover, the results disclose that there is remarkable difference in recognition between the left and right periocular regions of the same subject such that performance on the left side is better than that on the right side of the face.

\section{Discussion with Comparison with Previous Approaches}

Two metrics were used for performance evaluation in related previous works: the False Rejection Rate (FRR) and Guanine Accept Rate (GAR) [15]. The FRR is the proportion of genuine users who are rejected by the biometric system. In verification of a biometric system, the user will make claims of her/his identity and, therefore, the system must not reject enrolled user. That is, the number of false rejections (Equation Eq. (5)) must be kept at minimum.

$F R R=\left(\frac{\text { No.of rejected genuine }}{\text { Total no.genuine assessed }}\right) * 100$ 
TABLE IV. PERFORMANCE COMPARISON

\begin{tabular}{|l|l|}
\hline Approach & GAR (or Recognition accuracy) \\
\hline Ramaiah et al. [9] (140 subjects) & 73.20 \\
\hline Behera et al. [8] (209 subjects) & 83.12 \\
\hline Our approach (140 / 209 subjects) & $97.07 / 96.41$ \\
\hline
\end{tabular}

The GAR, on the other hand, is defined as the proportion of genuine users accepted by the system. It is given by Equation Eq. (6) [15]:

$G A R=100-F R R$

With this simple definition, it can be said that GAR is equivalent to the recognition accuracy. On account of this, the performance evaluation measure is actually the recognition accuracy.

Table IV points out that the approach which Behera et al. [8] followed produced better results than the approach followed by Ramaiah et al. [9]. The results of the current study prove to be very good relative to the results of these two studies (Table IV), with noticeable differences. The proposed PRS succeeded in almost all cases.

\section{CONCLUSIONS AND RECOMMENDATIONS FOR FUTURE WORK}

In this study, a PRS was proposed, developed, and tested. This system progresses in three steps: pre-processing, feature selection, and classification. The major contribution of this study is optimizing the periocular identity recognition process.

The feature extraction step is based on a combined LBP method and PCA. In this step, the features are extracted from the entire periocular image. In the meantime, the LBP method, which was originally developed for texture analysis, was employed to extract the features of the iris and periocular area as vectors. The study then applied PCA. Lastly, the k-NN algorithm was applied in the Matlab environment in order to determine the optimum value of $\mathrm{k}$ for the best recognition outcomes.

The study results were categorized according to three foundations. The first foundation was the number of the periocular images (images of the right or left side of the periocular area, or of both sides). The second foundation was region of the EMR spectrum (the VIS and NIR regions). The third foundation was the number of subjects whose images were employed in the experiments (140 and 209 subjects). The study results bring to surface that the proposed PRS best performed with 140 subjects when using the periocular images shot in the NIR region for pairs of pictures. The concomitant recognition accuracy was $98.21 \%$. Performance of the proposed PRS was compared with levels of performance of other systems. The comparison revealed that performance of the PRS proposed herein is better than levels of performance of other systems, with noticeable differences. Indeed, the proposed system gave the best recognition results in all the tested cases.

In view of the study results, the researchers suggest to testing the performance of the herein proposed PRS (i) on other dataset(s), (ii) in iris recognition, and (iii) using other classification methods like the ANN.

Lastly, periocular recognition can be further improved by considering clues such as eye shape and eye size.

\section{REFERENCES}

[1] Joke A. Badejo, Adekunle A. Akinrinmade, Emmanuel Adetiba, (2019)," Survey of Periocular Recognition Techniques", Journal of Engineering Science and Technology Review Vol.12 , No 5,pp 214 226.

[2] Kumari P., Seeja K.R. (2019), " Periocular biometrics: A survey", Elsevier B.V. on behalf of King Saud University.

[3] Fasca Gilgy Mary P., Sunitha Kency Paul P., Dheeba J., (2013)," Human Identification Using Periocular Biometrics", International Journal of Science, Engineering and Technology Research (IJSETR), Vol. 2, No. 5.

[4] Park U., Ross A., and Anil K. Jain, (2009) " Periocular Biometrics in the Visible Spectrum: A Feasibility Study", Theory, Applications and Systems (BTAS 09), Washington DC.

[5] Woodard D. L., Pundlik S., Miller P., Jillela R., and Ross A., (2010), "On the fusion of periocular and iris biometrics in non-ideal imagery", in Proceedings of International Conference on Pattern Recognition, pp. 201-204.

[6] Sharma A., Verma S., Vatsa M., Singh R., (2014), "On cross spectral periocular recognition", Image Processing (ICIP) 2014 IEEE International Conference on. IEEE, pp. 5007-5011.

[7] Nie L., Kumar A. and Zhan S., (2014), "Periocular Recognition Using Unsupervised Convolutional RBM Feature Learning", Proc. 22nd Intl. Conf. on Pattern Recognition, ICPR 2014, Stockholm, pp. 399-404.

[8] Behera S. S., Gour M., Kanhangad V., and Puhan N., (2017), "Periocular recognition in cross-spectral scenario", In IEEE IJCB, pp 681-687.

[9] Ramaiah N. P. and Kumar A., (2016), "On matching cross-spectral periocular images for accurate biometric identification," in 8th IEEE International Conference on Biometrics: Theory, Applications, and Systems (BTAS '16).

[10] Ramaiah N. P. and Kumar A., (2017), “Toward More Accurate Iris Recognition Using Cross-Spectral Matching”, IEEE Transactions on Image Processing, Vol. 26, No. 1, pp. 208-221.

[11] Ojala T., Pietikainen M., and Maenpaa T., (2002), "Multiresolution gray-scale and rotation invariant texture classification with local binary patterns," IEEE Transactions on Pattern Analysis and Machine Intelligence, Vol. 24, No. 7, pp. 971-987.

[12] Gautam D., (2014), "Facial expression detection using implemented PCA algorithm", International Journal of Computer Science and Technology Research, Vol. 2, No. 7, pp. 68-73.

[13] Chomboon K., Chujai P., Teerarassammee P., Kerdprasop K., Kerdprasop N., (2015). “An Empirical Study of Distance Metrics for kNearest Neighbor Algorithm”, The Proceedings of the 2nd Int. Conf. on Industrial Application Engineering 2015, 280-285.

[14] Burge MJ, Monaco MK (2009)," Multispectral iris fusion for enhancement, interoperability, and cross wavelength matching.", Proceeding of SPIE 7334, Algorithms and Technologies for Multispectral, Hyperspectral, and Ultraspectral Imagery XV, Vol. 7334. SPIE. pp 73341D-1-73341D-8.

[15] Shodhganga,https://shodhganga.inflibnet.ac.in/bitstream/10603/55920/1 1/11_chapter6.pdf Webpage last accessed 18/7/2020. 